\title{
INFECÇÃO POR CHLAMYDIA EM PACIENTES COM E SEM LESÖES INTRA-EPITELIAIS CERVICAIS
}

\author{
Micheline de lucena Oliveira, Melania Maria Ramos de Amorim*, Alex Sandro Rolland de Souza, lúcia Cristina Bezerra de Albuquerque, Aurélio \\ Antônio Ribeiro da Costa
}

Trabalho realizado pelo Instituto Materno-Infantil Prof. Fernando Figueira (IMIP), Recife, PE

\author{
*Correspondência: \\ Rua Neusa Borborema de \\ Souza, 300 - Santo Antônio \\ Campina Grande - PB \\ CEP 58103-313 \\ melamorim@uol.com.br
}

\begin{abstract}
RESUMO
OвıEтIVos. Determinar a frequência de infecção por Chlamydia trachomatis em pacientes com e sem lesões intra-epiteliais cervicais atendidas em ambulatório especializado no Recife (2007), e sua associaçãa com variáveis biológicas, demográficas, hábitos, características reprodutivas e clínico-ginecológicas.

Métodos. Realizou-se um estudo do tipo corte transversal, incluindo 70 mulheres (35 com alterações citológicas e 35 normais). Realizaram-se colposcopia, biópsia quando necessário e pesquisa para Chlamydia trachomatis por Imunofluorescência Direta. As variáveis analisadas foram idade, raça, procedência, escolaridade, estado civil, menarca, idade da primeira relação sexual, paridade, número de parceiros, corrimento, realização de citologia prévia, episódios de DST, eletrocauterização, método contraceptivo, antecedente familiar de câncer uterino, consumo alcoólico, tabagissmo, drogas ilícitas e imunossupressoras, resultado da citologia e infecção cervical por Chlamydia trachomatis. Para determinação da força da associaçãa, calculou-se a Razão de Prevalência (RP) e o intervalo de confiança 95\%, realizandose análise multivariada para controle das variáveis potencialmente confundidoras.

Resultados. A frequêencia de infecção por Chlamydia trachomatis foi significativamente maior em pacientes com alterações citológicas (80\% vs. 14,3\%), com uma RP de 5,60 (IC 95\% = 2,44 - 12,82). Analisando os fatores associados à infecção por Chlamydia , a única variável que persistiu significativamente associada após análise multivariada foi a história pregressa de DST (OR=63,47; IC 95\% = 13,93 - 289,09).

Conclusã̃o. A presença da Chlamydia trachomatis está associada às alterações citológicas da cérvice uterina, e a história pregressa de DST deve ser valorizada no tratamento e seguimento clínico destas pacientes.

Unitermos: Chlamydia trachomatis. Infecções por Chlamydia. Neoplasia intra-epitelial cervical. Técnica direta de fluorescência para anticorpo.
\end{abstract}

\section{INTRODUÇão}

A infecção por Chlamydia trachomatis tem sido reconhecida como um dos maiores problemas de saúde pública 1,2,3. Dados da Organização Mundial de Saúde (OMS) mostram que as doenças sexualmente transmissiveis são a segunda enfermidade que mais acomete as mulheres entre 15 e 44 anos nos países em desenvolvimento ${ }^{1,2}$. Em relação à Chlamydia trachomatis, ocorrem cerca de 50 milhões de casos novos no mundo por $a n 0^{3}$, e no Brasil não existem estudos documentando a situação global da infecção pela Chlamydia trachomatis. Estão disponíveis apenas estudos isolados em populações específicas, mas que mostram a importância dessa infecção silenciosa em nosso meio ${ }^{2,4}$.

Na maioria das mulheres (70\% a 75\%) e em mais de $50 \%$ dos homens essas infecções cursam de forma assintomática ${ }^{1,3,5}$. Assim, esse microorganismo pode ser considerado bem adaptado ao ser humano, uma vez que consegue multiplicar-se sem causar respostas exacerbadas do organismo, daí as dificuldades para o seu diagnóstico 23,5.

Esta peculiaridade retarda o tratamento, permitindo que os casos de infecção genital se propaguem ao trato genital superior, causando endometrites e salpingites ${ }^{3,5,6}$. Hoje, a infecção pela Chlamydia trachomatis é considerada como uma das Doenças Sexualmente Transmissiveis (DST) mais freqüentes em todo o mundo 1,2,3,7.

Em 2005, 976.445 casos de infecção genital por Chlamydia trachomatis foram relatados ao Centers for Disease Control and Prevention (CDC) nos EUA, correspondendo a uma taxa de 332.5 casos por 100.000 habitantes. A prevalência em mulheres nos diversos estados variou entre 3,1\% a I4,5\% (mediana de 9,2\%), sendo maior ainda em adolescentes, com mediana de positividade para Chlamydia de $14,2 \%$ (variando de $3,7 \%$ a $33,7 \%)^{7}$.

Nos serviços públicos brasileiros, são raros os locais oferecendo sistematicamente a pesquisa da Chlamydia. Nos serviços privados, normalmente só se pesquisa essa infecção em casos sintomáticos ou quando um dos parceiros sexuais está acometido. Mesmo nessas situações, a pesquisa da Chlamydia trachomatis ainda não faz parte da rotina da maioria dos ginecologistas, urologistas ou médicos que atendem DST, apesar da sua importância e sua possível relação com o câncer de colo uterino ${ }^{2,4.8}$. 
agente etiológico nas neoplasias do trato genital inferior da mulher é, segundo evidências clínicas, biomoleculares e epidemiológicas, o papilomavírus humano (HPV) que irá agir no alvo suscetível: as células metaplásicas imaturas ou células basais do epitélio pavimentoso $0^{9,10}$. Entretanto, são necessários co-fatores de promoção complementares favorecedores da instalação do agente HPV são: idade da paciente ${ }^{12}$, início precoce das relações sexuais, multiplicidade de parceiros ${ }^{12}$, primeira gestação na adolescência ${ }^{12}$, multiparidade ${ }^{10,11}$, antecedente de doenças sexualmente transmissíveis (DST)", incluindo-se a Chlamydia trachomatis, uso de contraceptivos hormonais orais ${ }^{13}$, tabagismo, ${ }^{14}$ déficit nutricional e imunológico (como pacientes HIV-positivas) ${ }^{15,16}$, além de fatores genéticos ${ }^{15}$.

A infecção persistente por Chlamydia trachomatis tem papel facilitador na carcinogênese cervical, por meio das heat shoch proteins 60(HSP60). Essas proteínas sintetizadas pelas Chlamydia trachomatis têm ação anti-apoptótica durante a infecção persistente, facilitando a atuação das oncoproteínas em células simultaneamente infectadas por HPV de alto risco (HPV tipo 16, 18 ) $^{16}$. Entretanto, a magnitude da associação entre Chlamydia trachomatis e lesões intra-epiteliais cervicais persiste por ser esclarecida.

Neste contexto, insere-se nossa proposta de determinar a freqüência da infecção por Chlamydia trachomatis nas pacientes com e sem lesões precursoras do câncer cervical em um serviço público da cidade do Recife e analisar as variáveis biológicas, demográficas, hábitos, características clínico-ginecológicas e reprodutivas associados à infecção por Chlamydia trachomatis.

\section{Métodos}

Realizou-se um estudo transversal de base populacional para determinar a freqüência da infecção por Chlamydia trachomatis nas pacientes com e sem lesões precursoras do colo uterino, atendidas no Ambulatório Especializado da Mulher da Prefeitura Municipal do Recife, durante o período de novembro de 2006 a abril de 2007. Este ambulatório é referência na atenção à saúde da mulher e atende ao III Distrito- U.S.- 129, com população total estimada de 308.063 habitantes ( Fonte : IBGE - Censo 2005), com uma média de atendimento de 1.787 mulheres / mês no ano de 2005.

A população de estudo foi composta por 70 mulheres selecionadas através da demanda livre e espontânea, com citologia oncótica realizada nas redes credenciadas municipais e/ou estaduais, apresentando ou não lesões de baixo e alto grau/ ASCUS/AGUS para câncer de colo uterino com validade de um ano e que não tivessem sido submetidas a tratamento prévio do colo uterino nos últimos seis meses; foram excluídas do estudo as mulheres HIV-positivas e aquelas que não aceitaram realizar sorologia para HIV. Não se encontrou nenhuma paciente em uso de drogas imunossupressoras.

O tamanho da amostra foi calculado no software EPI-INFO 3.3.2, utilizando-se o programa STATCALC, com base em dados de prevalência da literatura. Embora vários autores tenham relatado prevalências mais elevadas, optamos pelo estudo de Golijow et al. $(2005)^{17}$ realizado na Argentina, já que a população tem traços aparentemente semelhantes (país localizado na América do Sul), para avaliar a prevalência da Chlamydia trachomatis nas pacientes com citologias oncóticas normais e anormais. Neste estudo foram analisadas 279 mulheres, e observou-se que a prevalência por Chlamydia trachomatis foi de I I\% nas pacientes com citologias oncóticas normais ao passo que nas pacientes com citologias oncóticas anormais com lesão de baixo e alto grau / ASCUS houve um incremento para 47\%. Considerando-se infinito o tamanho da população, com uma precisão de 5,0 \%, um nível de confiança de $95 \%$, um poder de $80 \%$, seria necessário um tamanho da amostra de 58 mulheres. Para compensar possíveis perdas, este número foi aumentado para 70 mulheres (aproximadamente 20\%), sendo 35 com citologias oncóticas normais e 35 com citologias oncóticas anormais (lesões precursoras de baixo e/ou alto grau / ASCUS).

O estudo foi previamente aprovado pelo Comitê de Ética em Pesquisa do Instituto Materno-Infantil Prof. Fernando Figueira e todas as pacientes incluídas concordaram em participar, assinando o Termo de Consentimento Livre e Esclarecido.

Durante 0 atendimento foram realizados preenchimento de prontuário, colposcopia, biópsias (se necessário) e cultura do conteúdo vaginal e endocervical com pesquisa para Chlamydia trachomatis por imunofluorescência direta (IMF-Direta), solicitando-se o teste de HIV para as pacientes que não o tivessem realizado dentro do prazo de seis meses. Após o atendimento, foi agendado o retorno para tratamento e acompanhamento de cada caso.

As variáveis de análise estudadas foram idade, raça, procedência, escolaridade, estado civil, menarca, idade da primeira relação sexual, paridade, número de parceiros, história de corrimento, realização prévia do exame de Papanicolaou, episódios prévios de DST (conforme informaçãa das pacientes), eletrocauterização, método contraceptivo, antecedente familiar de câncer uterino/grau de parentesco, consumo de álcool, tabagismo, consumo de drogas ilícitas, resultado da citologia e infecção cervical por Chlamydia trachomatis.

Consideramos como citologia normal as citologias com células escamosas e/ou endocervicais sem anormalidades; com metaplasia escamosa e/ou com alterações inflamatórias; e como citologias anormais as que apresentavam diagnóstico de lesão de baixo grau, lesão de alto grau, carcinoma escamoso, atipias de células escamosas de significado indeterminado e atipias glandulares de significado indeterminado.

Infecção por Chlamydia trachomatis foi considerada presente quando o resultado da Imunofluorescência Direta era positivo, ou seja, a presença de pelo menos cinco corpos clamidianos, ou se número menor, quando não havia dúvida de se tratar de Chlamydia (e não de artefato). Considerou-se negativa a ausência de corpos clamidianos, mas com presença de células epiteliais com formas geométricas mais ou menos alongadas, garantindo a qualidade da amostra ${ }^{18}$ e seguindo as especificações dos kits Chlamydia Direct IF, do Fabricante BioMérieux. Foram rejeitadas cinco amostras hemorrágicas e uma purulenta, coletando-se posteriormente nova amostra das mesmas pacientes.

A coleta da amostra consistiu em colocar um espéculo (sem lubrificante) na vagina (das mulheres não menstruadas, sem utilizar duchas e cremes vaginais na véspera e três dias de abstinência sexual), retirar o excesso de muco cervical, e após inserir swab de algodão indicado no canal endocervical até a ponta do swab não ser mais visível, 
rodar por alguns segundos, retirar evitando o contato com a parede vaginal e colocar em lâmina citológica devidamente identificada. Após secar completamente à temperatura ambiente, esta lâmina foi fixada com acetona, por imersão, durante 10 min. Após evaporação completa do fixador, as lâminas foram acondicionadas em recipientes próprios e encaminhadas ao laboratório, sendo processadas no máximo 24h após a coleta e a maioria delas nas primeiras horas após a coleta.

Pacientes com IMF-Direta para Chlamydia positiva receberam azitromicina na dose de I grama por via oral, em dose única. As pacientes com citologia alterada foram acompanhadas de acordo com as normas do Ministério da Saúde ${ }^{15}$.

Os dados foram armazenados, revisados e analisados no software Epi-Info versão 3.4. I, com dupla entrada. Para determinação da força da associação entre as diversas variáveis e a infecção por Chlamydia trachomatis, foi calculada a razão de prevalência (RP) e o seu intervalo de confiança a 95\%, realizando-se análise multivariada para controle das variáveis potencialmente confundidoras. No modelo de regressão logística múltipla, entraram todas as variáveis independentes (preditoras) e a variável dependente (infecção por Chlamydia trachomatis), recodificadas binariamente como $\mid=\operatorname{sim}$ e $0=$ não. As variáveis foram progressivamente retiradas do modelo adotando-se inicialmente o nível de significância de 10\%, até que no modelo final persistiram apenas as variáveis associadas com a infecção por Chlamydia trachomatis , considerando-se o nível de significância de 5\%.

\section{Resultados}

A freqüência de infecção por Chlamydia trachomatis foi significativamente maior em pacientes com alterações citológicas (80\% vs. I4,3\%), com uma RP de 5,60 (IC 95\% = 2,44 - 12,82), como se observa na Tabela I.

Analisando-se os fatores associados à infecção por Chlamydia trachomatis, observou-se associação de infecção por Chlamydia com as variáveis procedência ( $100 \%$ em pacientes de zona rural, 44,8\% em pacientes de zona urbana; $R P=2,23$; IC 95\% = I,7I-2,9I) e escolaridade $59 \%$ em mulheres com oito ou mais anos de escolaridade e $32,3 \%$ naquelas com menos de oito anos de estudo; $R P=1,83$; IC $95 \%=1,03-3,24)$. Não houve associação entre infecção por Chlamydia e as variáveis idade, cor, situação marital, consumo de álcool e de drogas ilícitas (Tabela 2).

Por outro lado, dentre as variáveis reprodutivas e clínico-ginecológicas, observou-se associaçãao estatisticamente significante da infecção por Chlamydia com história de corrimento (80,6\% em pacientes com história positiva e 11,8\% naquelas com história negativa; $\mathrm{RP}=6,85 ; \quad I C 95 \%=2,69-17,4)$ e episódios pregressos de DST (87,5\% em mulheres com história de DST e 13,2\% em mulheres sem história de DST; RP=6,65; IC 95\%=2,9|-|5,2I). Não se encontrou associação significativa da infecção por Chlamydia com idade da menarca, idade da primeira relação sexual, paridade, número de parceiros sexuais, realização de citologia oncótica prévia, história de eletrocauterização, uso de método contraceptivo hormonal e antecedente familiar de câncer de colo uterino (Tabela 3).

Depois de efetuado o controle das variáveis potencialmente confundidoras através de análise multivariada (regressão logística

\begin{tabular}{|c|c|c|c|c|c|c|}
\hline \multirow[t]{3}{*}{$\begin{array}{l}\text { Resultado da } \\
\text { citologia }\end{array}$} & \multicolumn{4}{|c|}{$\begin{array}{c}\text { Infecção por } \\
\text { Chlamydia trachomatis }\end{array}$} & \multirow[t]{3}{*}{ RP } & \multirow[t]{3}{*}{ IC 95\% } \\
\hline & \multicolumn{2}{|c|}{ Sim } & \multicolumn{2}{|c|}{ Não } & & \\
\hline & $\mathbf{N}$ & $\%$ & $\mathrm{~N}$ & $\%$ & & \\
\hline $\begin{array}{l}\text { Alterada } \\
\text { Normal } \\
\text { TOTAL }\end{array}$ & $\begin{array}{c}28 \\
5 \\
33\end{array}$ & $\begin{array}{l}80,0 \\
14,3 \\
47 \mid\end{array}$ & $\begin{array}{c}7 \\
30 \\
37\end{array}$ & $\begin{array}{l}20,0 \\
85,7 \\
529\end{array}$ & $\begin{array}{l}5,60 \\
1,00\end{array}$ & $2,44-12,82$ \\
\hline
\end{tabular}

$c^{2}=30,33 p=0,000000$

Tabela 2 - Frequêencia da infecção por Chlamydia trachomatis de acordo com as variáveis biológicas, demográficas e hábitos.

\begin{tabular}{|c|c|c|c|c|c|c|}
\hline \multirow[t]{4}{*}{ Variáveis } & \multicolumn{4}{|c|}{ Infecção por } & \multirow[t]{3}{*}{$\mathbf{R R}$} & \multirow[t]{2}{*}{ IC 95\% } \\
\hline & \multicolumn{4}{|c|}{ Chlamydia trachomatis } & & \\
\hline & \multicolumn{2}{|c|}{ Sim } & \multicolumn{2}{|c|}{ Não } & & \multirow[b]{2}{*}{$\%$} \\
\hline & \multicolumn{2}{|c|}{$\mathbf{N}$} & \multicolumn{2}{|c|}{$\%$} & \multirow[t]{2}{*}{$\mathbf{N}$} & \\
\hline \multicolumn{6}{|l|}{ Idade } & \\
\hline $\begin{array}{l}\leq 25 \text { anos } \\
>25\end{array}$ & $\begin{array}{c}7 \\
26\end{array}$ & $\begin{array}{l}43,8 \\
48,1\end{array}$ & $\begin{array}{c}9 \\
28\end{array}$ & $\begin{array}{l}56,3 \\
51,9\end{array}$ & $\begin{array}{l}0,91 \\
1,00\end{array}$ & $0,49-1,70$ \\
\hline \multicolumn{7}{|l|}{ Cor } \\
\hline Negra/parda/Amarela & 21 & 50,0 & 21 & 50,0 & 1,17 & $0,69-1,97$ \\
\hline Branca & 12 & 42,9 & 16 & 57,1 & 1,00 & \\
\hline \multicolumn{7}{|l|}{ Procedência } \\
\hline & 3 & 100,0 & - & - & 2,23 & $|, 7|-2,9 \mid$ \\
\hline Urbana & 30 & 44,8 & 37 & & 1,00 & \\
\hline \multicolumn{7}{|l|}{$\begin{array}{l}\text { Escolaridade } \\
\text { (anos) }\end{array}$} \\
\hline$\geq 8$ anos & 23 & 59,0 & 16 & 41,0 & 1,83 & $1,03-3,24$ \\
\hline$<8$ anos & 10 & 32,3 & 21 & 67,7 & 1,00 & \\
\hline \multicolumn{7}{|l|}{ Situação marital } \\
\hline Com companheiro & 15 & 44,1 & 19 & 55,9 & 0,88 & $0,54-1,45$ \\
\hline Sem companheiro & 18 & 50,0 & 18 & 50,0 & 1,00 & \\
\hline \multicolumn{7}{|l|}{ Consumo de fumo } \\
\hline Sim & 4 & 57,1 & 3 & 42,9 & 1,24 & $0,62-2,49$ \\
\hline Não & 29 & 46,0 & 34 & 54,0 & 1,00 & \\
\hline \multicolumn{7}{|l|}{ Consumo de álcool } \\
\hline & 11 & 45,8 & 13 & 54,2 & 0,96 & $0,56-1,63$ \\
\hline Não & 22 & 47,8 & 24 & 52,2 & 1,00 & \\
\hline \multicolumn{7}{|l|}{$\begin{array}{l}\text { Consumo de } \\
\text { drogas ilícitas }\end{array}$} \\
\hline Sim & I & 33,3 & 2 & 66,7 & 0,70 & $0,14-3,53$ \\
\hline Não & 32 & 47,8 & 35 & 52,2 & 1,00 & \\
\hline
\end{tabular}

múltipla), a única variável que persistiu significativamente associada com a infecção por Chlamydia diagnosticada por imunofluorescência foi a história pregressa de DST (OR=63,47; IC 95\% = 13,93 - 289,09). Nem o resultado da citologia nem as variáveis procedência, corrimento e escolaridade apresentaram associação significante com a infecção por Chlamydia no modelo final. 


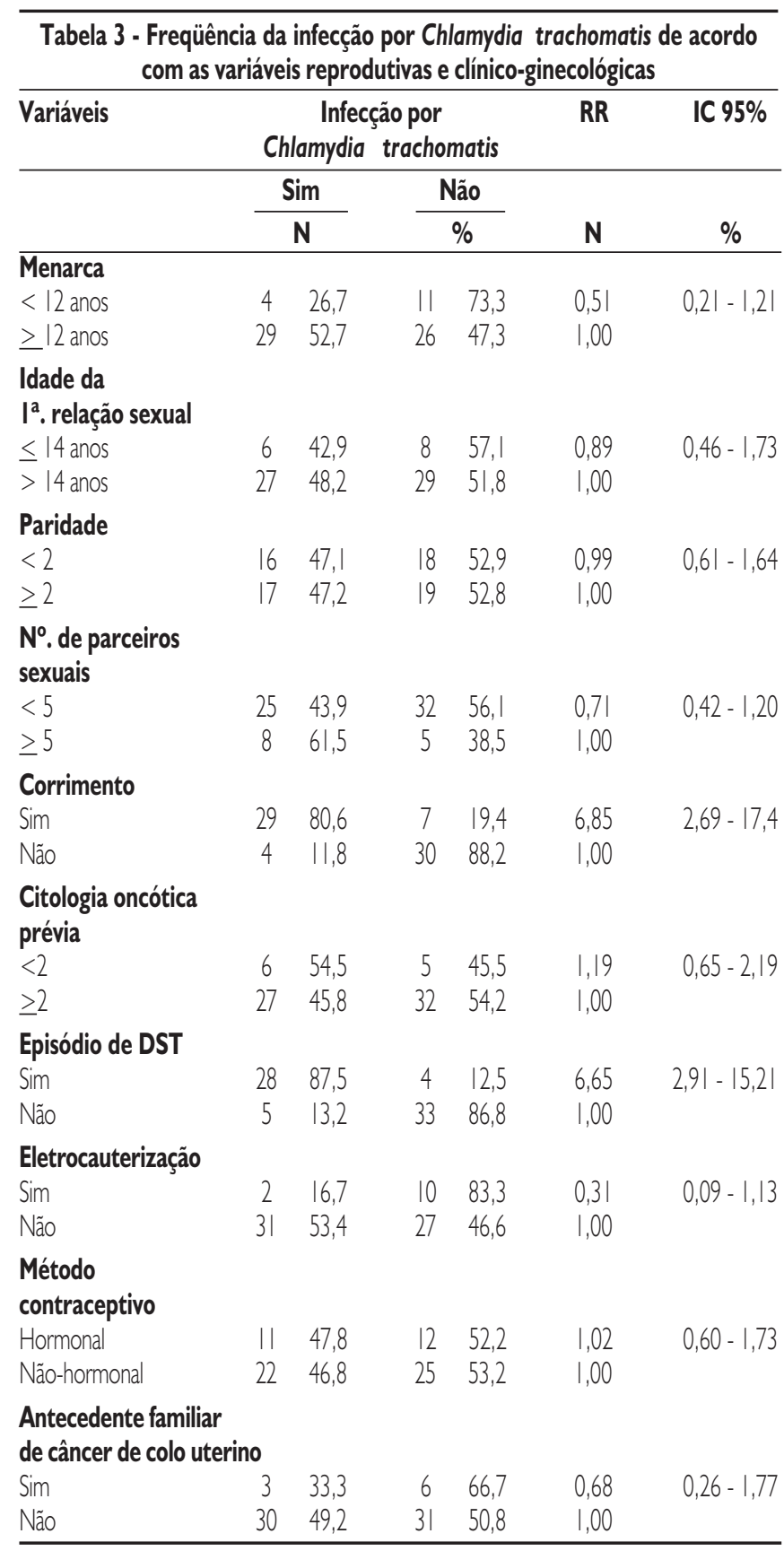

\section{Discussão}

No presente estudo, evidenciamos uma prevalência de infecção por Chlamydia trachomatis em pacientes com citologia oncótica normal de $14,3 \%$ e em pacientes com citologia alterada de $80 \%$. O principal fator associado à presença de infecção por Chlamydia foi a história pregressa de DST, que persistiu depois de análise multivariada para controle das variáveis potencialmente confundidoras.

A prevalência real da infecção por Chlamydia trachomatis em nosso meio é desconhecida, sendo necessários estudos populacionais para sua avaliação. A prevalência varia de acordo com a população estudada e o método diagnóstico adotado ${ }^{2,4}$. Teles et al., em 2007, avaliaram 407 mulheres do Ambulatório de Planejamento Familiar, em Campinas e encontraram uma prevalência de $6,6 \%$, tendo sido utilizado como método diagnóstico a técnica de imunofluorescência direta ${ }^{13}$. Essa prevalência foi bem inferior à que encontramos no presente estudo, o que pode se dever tanto ao fato de ter sido realizada em pacientes assintomáticas procurando acesso a métodos contraceptivos, como a reais diferenças na população estudada e nos testes utilizados para diagnóstico.

Existem diversas metodologias que podem ser utilizadas para a detecção da Chlamydia trachomatis. A escolha de um determinado método deve levar em consideração a prevalência da infecção na população que vai ser examinada, para que se defina uma metodologia com sensibilidade, especificidade e reprodutibilidade compatíveis ${ }^{17}$. Além disso, é importante que se considere o custo-benefício do teste, as dificuldades para a sua execução, os equipamentos, infra-estrutura laboratorial e o tempo necessário para a liberação dos resultados ${ }^{18}$.

Os métodos mais amplamente utilizados são os de detecção do antígeno da Chlamydia trachomatis na amostra clínica. Método diagnóstico factível e com resultados aceitáveis, a imunofluorescência direta consiste na identificação dos corpúsculos elementares em material endocervical, através de anticorpos monoclonais fluorescentes, identificados através de microscópio ótico ${ }^{5,18}$. Esta técnica utiliza um ou mais anticorpos monoclonais conjugados com moléculas fluorescentes contra a principal proteína da membrana externa da Chlamydia trachomatis. A sua sensibilidade atinge $95 \%$ e a especificidade até $100 \% 5,18$.

Existem também outros testes laboratoriais para a detecção direta da Chlamydia trachomatis como a cultura, o enzimaimunoensaio (ELISA), a sonda de DNA, as técnicas de amplificação de ácidos nucléicos e os testes de diagnóstico indireto através da pesquisa de anticorpos. As técnicas sorológicas mais comuns, como a fixação do complemento e a imunofluorescência indireta, são freqüentemente recomendadas para estudos epidemiológicos e infecções sistêmicas, em que os títulos de anticorpo lgG são freqüentemente elevados (maiores ou iguais a $1: 256)^{5,18}$. Entretanto, não são recomendadas para o diagnóstico de infecções urogenitais por causa da freqüência de exposiçãa aos sorotipos da Chlamydia trachomatis e pela ocorrência de reações cruzadas com outras espécies 5.18 .

A cultura permite o cultivo de várias linhagens celulares, sendo mais utilizadas as células de McCoy distribuídas em monocamadas sobre microplacas. A vantagem da cultura é a baixa probabilidade de contaminação e a preservação do microrganismo para estudos adicionais, como o teste de suscetibilidade à terapia antimicrobiana e genotipagem. A desvantagem é a necessidade de infra-estrutura de laboratório muito onerosa e trabalhosa, além de exigir cuidados na conservação da amostra (microrganismos viáveis). Sua especificidade é de 100\%, porém sua sensibilidade, mesmo em laboratórios de excelência, é de $80 \%$, 18 .

Codes et al., utilizando a técnica de amplificação do DNA na urina para diagnóstico, evidenciaram uma prevalência dessa infecção de I I,4\% em uma clínica de Planejamento Familiar no Brasil (Bahia) ${ }^{4}$. Nesse estudo, $60 \%$ das mulheres eram assintomáticas e não usuárias de métodos anticonceptivos; ao passo que as usuárias de métodos de 
planejamento familiar apresentaram uma prevalência de 4,5\% a I2,4\% para a Chlamydiat. Esses resultados se assemelham ao que encontramos em pacientes com citologia oncótica negativa, possivelmente porque havia semelhanças entre a população (Nordeste).

Em outro estudo do mesmo grupo, realizado em $2006^{8}$, verificouse uma variação na prevalência de acordo com o nível de instrução e o nível socioeconômico, que variou de I I,4\% (geral) a 17,1\% (nível de instrução secundário) e de 12,9\% (nas comunidades pobres). Em nosso estudo, observamos que a infecção por Chlamydia trachomatis foi maior ( $59 \%$ vs. 32,3\%) nas pacientes com maior escolaridade e nas de procedência rural (I00\% vs. 44,8\%). Este incremento no risco possivelmente deve-se ao fato de que as pacientes mais instruídas tendem a se cuidar mais e procuram centros de referência para diagnóstico e tratamento.

No México, através de um estudo transversal multicêntrico, encontrou-se uma prevalência de Chlamydia através da pesquisa da IgG de II,4\% e IgA de 4,4\% ${ }^{19}$; a infertilidade por dano tubário foi de $8,4 \%$ ( $\operatorname{lgG})$ e I,4\% $(\lg A)^{19}$, ao passo que outros estudos em Londres demonstraram uma prevalência de 2,6\% ${ }^{20}$. Em Bogotá, Colômbia, observou-se uma prevalência de Chlamydia de 5\%21. Todos esses resultados aproximam-se das taxas encontradas em nosso estudo nas pacientes com citologia oncótica negativa, possivelmente por semeIhanças entre a população (América do Sul).

Em relação à associação entre infecção por Chlamydia trachomatis e lesões precursoras do colo uterino, nosso estudo difere dos resultados encontrados por outros autores. No estudo de Edelman et al. ${ }^{22}$, não se observou impacto da prevalência de Chlamydia sobre as citologias oncóticas anormais; Reesink-Peters et al. ${ }^{23}$ também não encontraram associação da infecção por Chlamydia trachomatis com a gravidade da lesão neoplásica e a velocidade de progressão da neoplasia cervical ${ }^{23}$. Entretanto, essa diferença pode ter ocorrido devido ao método utilizado para o diagnóstico e/ou ao local de coleta destas pacientes, porque outros estudos com metodologia diferente encontraram associação significativa entre infecção por Chlamydia trachomatis e lesões intra-epitelias cervicais.

Em um estudo comparativo realizado na Argentina, Golijow et al. ${ }^{17}$ determinram a prevalência do papilomavírus (HPV) e Chlamydia trachomatis em 279 mulheres pelo método da pesquisa de ácidos nucléicos (DNA), destas 79 com citologia oncótica normal e 200 com anormal. Observou-se que a prevalência de Chlamydia trachomatis foi de I I\% nas pacientes com citologia oncótica normal, ao passo que nas pacientes com citologia oncótica anormal com lesão de alto grau houve um incremento para $47 \%$ e de $20 \%$ nos casos de carcinoma escamoso. Por outro lado, a presença do HPV variou de $30 \%$ nas pacientes com citologia oncótica normal até 99\%-100\% nas pacientes com citologia oncótica anormal. Desta forma, demonstrou-se uma forte associação com as lesões precursoras do câncer cervical de baixo e alto grau ${ }^{17}$.

Outro estudo realizado no Brasil e nas Filipinas mostrou que a Chlamydia foi um possível co-fator do HPV na etiologia do carcinoma cervical invasivo (OR: 2, I; IC 95\%= I, I-4,0), com resultados similares em ambos países ${ }^{24}$. Koskela et al. ${ }^{25}$ mostraram uma associação da Chlamydia trachomatis com o desenvolvimento do carcinoma escamoso do colo uterino. Esses achados semelhantes aos nossos podem ser explicados pela possibilidade de a infecção persistente por
Chlamydia trachomatis ter um papel facilitador na carcinogênese cervical, através das heat shoch proteins 60 , que possuem ação anti-apoptótica, facilitando a atuação das oncoproteínas em células simultaneamente infectadas por HPV de alto risco (HPV tipo 16, 18) 16. $^{16}$.

Em um estudo realizado em Beirute, 2002, analisou-se a relação causal entre o HPV e a Chlamydia em I2I mulheres com citologia normal ou alterada, observando-se uma freqüência de Chlamydia significativamente maior em mulheres não-fumantes, maior paridade, nas pacientes com citologias oncóticas anormais (lesões de baixo e alto grau) e maior número de parceiros ${ }^{26}$. Também se observou associação da infecção por Chlamydia com a gravidade da lesão neoplásica e a velocidade de progressão da neoplasia cervical. ${ }^{26}$

Em nosso estudo, apesar da associação evidenciada entre infecção por Chlamydia e lesão intra-epitelial cervical, não evidenciamos associação de infecção por Chlamydia e a maior parte dos fatores de risco acima descritos, à exceção da história de infecção por DST. Embora escolaridade maior que oito anos, procedência rural e presença de corrimento genital tenham surgido como fatores associados à infecção por Chlamydia na análise bivariada, na análise multivariada 0 único fator persistindo associado à Chlamydia foi a história pregressa de DST. Apesar de essa variável ter sido coletada apenas a partir da informação das participantes, sem realização de exames comprobatórios, acreditamos que de fato reflita a ocorrência de DST no passado, sendo possíveis vieses de recordação e resultados falsonegativos, porém com reduzida probabilidade de falso-positivos.

Sabe-se que a Chlamydia necessita do crescimento intracelular obrigatório utilizando-se do aparato enzimático da célula do hospedeiro para a produção de ATP e a sua replicação invariavelmente determina a morte desta célula, sendo sempre considerada patogênica ${ }^{2.5}$. Atualmente, o mecanismo pelo qual esse patógeno induz a inflamação e dano tecidual é apenas parcialmente conhecido. Sabe-se que há uma abundante resposta imune à infecção pelas Chlamydia s, isso tanto em relação à imunidade humoral como à mediada por células ${ }^{9}, 16.0$ processo infeccioso depende basicamente da capacidade de defesa do organismo e da agressividade do microorganismo. $\mathrm{O}$ embate entre estes dois fatores pode determinar o bloqueio do processo na sua fase inicial ou o desencadeamento do processo infeccioso de modo ameno ou com graus variáveis da gravidade clínica 2,9,16

Há evidências de que pelo menos parte do dano é decorrente de reações de hipersensibilidade tardia ${ }^{16}$. Um provável antígeno relacionado à sensibilização é a proteína HSP60, pertencente à classe das proteínas de choque térmico (PCT) ou, heat shock proteins (HSP). ${ }^{16}$ Essas proteínas sintetizadas pelas Chlamydia trachomatis possuem grande semelhança com as PCT humanas, assim as proteínas clamídianas poderiam sensibilizar os linfócitos a responderem de forma cruzada com as PCT humanas, e a expressão desses antígenos nas células dos tecidos do hospedeiro poderia induzir uma resposta imunológica contra as células expressoras, resultando na destruição dessas células ${ }^{16}$. Percebe-se também que a concentração de anticorpos séricos contra PCT clamidianas guarda correlação com a intensidade do dano. ${ }^{16}$ Sendo assim, quanto maior o dano causado à célula, justificarse-ia a associação da Chlamydia com as lesões precursoras da cérvice, bem como com fatores de risco como tabagismo ${ }^{14}$ e nível de instrução ${ }^{12}$. Por outro lado, a história pregressa de qualquer DST é um 
indicador importante de variáveis ligadas ao comportamento sexual, incluindo a possibilidade de contágio tanto pela Chlamydia trachomatis como pelo HPV. Daí, possivelmente, a explicação de essa variável (infecção por DST) ser a única que persistiu associada à infecção por Chlamydia trachomatis na análise multivariada.

\section{Conclusão}

Encontrou-se associação entre infecção por Chlamydia trachomatis e alterações citológicas da cérvice uterina, enquanto o principal fator associado com a infecção por Chlamydia foi a história pregressa de DST. Acreditamos que essas informações devem ser valorizadas para o tratamento e seguimento clínico das mulheres com lesões intra-epiteliais cervicais, podendo auxiliar na implementação das estratégias de prevenção e diagnóstico precoce das lesões precursoras do colo uterino.

São necessários estudos amplos, nacionais, visando a determinar a real prevalência da infecção por Chlamydia trachomatis em nosso meio, e aprofundar a discussão que ora se inicia, sobre os fatores associados à presença desta infecção e as implicações de sua associação com as lesões intra-epiteliais cervicais.

Enquanto esses grandes estudos populacionais não estão disponíveis, acreditamos que os presentes resultados justificam a proposta de tratamento em massa para Chlamydia trachomatis de todas as pacientes apresentando lesão intra-epitelial cervical em nosso meio. Por outro lado, como o fator mais importante associado à infecção por Chlamydia foi a história pregressa de DST, também se poderia aventar a sugestão de realizar o tratamento para Chlamydia trachomatis em todas as mulheres referindo episódios prévios de DST, mesmo sem ter realizado exame para detecção da Chlamydia trachomatis. Estudos posteriores devem ser conduzidos para testar a efetividade desse tipo de intervenção e acompanhamento da taxa de regressão das lesões intra-epiteliais depois do tratamento.

\section{Conflito de interesse: Não há}

\section{SUMMARY}

\section{Chlamydia infection in patients With and Without CERVICAL INTRA-EPITHELIAL LESIONS}

OBIECTINES. To determine the frequency of Chlamydia trachomatis infection in patients with and without intraepithelial cervical lesions attended at specialized ambulatory in Recife (2007) and its association with biologic and demographic variables, habits, reproductive, clinical and gynecologic characteristics.

METHODS. A cross-sectional study was conducted including 70 women (35 with cytological cervical lesions and 35 with normal smears). Colposcopy and biopsy when necessary were performed and Chlamydia infection was determined by direct immunofluorescence. Analysis variables were age, race, precedence, schooling, marital status, menarche, age at first sexual intercourse, parity, number of sexual partners, vaginal discharge, previous Pap smear, STD episodes, cervical cauterization, contraceptive methods, familial antecedents of cervical cancer, alcohol intake, use of illicit and immunosuppressive drugs, Papanicolaou result and cervica/Chlamydia / infection. To determine the strength of association prevalence ratio (PR) and its 95\% confidence interval were calculated. Multivariate analysis was performed for controlling potentially confounding variables.

RESULTS. the frequency of Chlamydia / infection was significantly greater in patients with intraepithelial cervical lesions $(80.0 \%$ vs. $14.3 \%$; $P R=5.60 ; 95 \% \mathrm{Cl}=2,44-12,82)$. When the factors associated with Chlamydia I infection were analyzed, the only variable that remained significantly associated after multivariate analysis was previous episode of $\operatorname{STD}(O R=63,47 ; 95 \% \mathrm{Cl}=13,93-289,09)$.

CONCLUSION. Chlamydia trachomatis infection is associated with intraepithelial cervical lesions and history of STD should be considered for treatment and follow-up of these patients. [Rev Assoc Med Bras 2008; 54(6): 506-12]

KEY Words: Chlamydia trachomatis. Chlamydia I infections. Cervical intraepithelial neoplasia. Fluorescent antibody technique, direct.

\section{REFERÊNCIAS}

I. World Health Organization 2005. Sexually transmitted and other reproductive tract infections. Geneva: World Health Organization; 2005 [cited 2007 Nov 20]. Available from: http://www.who.int/reproductivehealth/publications/rtis_gep/rtis_gep.pdf.

2. Ministério da Saúde. SPC-CNDST/Aids. Manual de controle de DST. $3^{\text {a }}$ ed. Brasília(DF); 1999. [citado 20 Nov 2007]. Disponível em: http:// www.acemfc.org.br/modelol/down/manual_controle_dst.pdf.

3. World Health Organization. Department of HIV/AIDS. Global prevalence and incidence of selected curable sexually transmitted infections. Geneva: World Health Organization, 200 I. [cited 2007 Nov 20]. Available from: http://www.who.int/docstore/hiv/GRSTI/003.htm.

4. Codes JS, Cohen DA, Melo NA, Santos AB, Codes JJG, Silva Jr JC, et al. Detecção de doenças sexualmente transmissíveis em clínica de planejamento familiar da rede pública no Brasil. Rev Bras Ginecol Obstet. 2002;24: 101-6.

5. Frias MCAA, Pereira CFA, Pinheiro VMS, Pinheiro MS, Rocha CF. Freqüência de Chlamydia trachomatis, Ureaplasma urealyticumNetto e Mycoplasma hominis na endocérvice de mulheres no menacme. DST J Bras Doenças Sex Transm. 200 I; 13: 5-22.

6. Melles HHB, Colombo S, Linhares IM, Siqueira LFG. Avaliação de parâmetros para o diagnóstico laboratorial de infecção genital feminina pela Chlamydia trachomatis. Rev Soc Bras Med Trop. 2000;33:355-61.

7. Center for Disease Control and Prevention. Sexually transmitted disease surveillance 2005 supplement. In: Chlamydia Prevalence Monitoring Project Annual Report. Atlanta; 2005. [cited 2007 Oct 8]. Available from: http://www.cdc.gov/std/Chlamydia 2005/CTSurvSuppComplete.pdf.

8. Codes JS, Cohen DA, Melo NA, Teixeira GG, Leal AS, Silva TJ, et al. Detecção de doenças sexualmente transmissíveis e não clínicos na cidade de Salvador Bahia, Brasil. Cad Saúde Pública. 2006;22:325-34.

9. Paavonem J. Chlamydia trachomatis and cancer. Sex Transm Infect. 2001;77:154-6.

10. Finan RR, Tamim H, Almawi WY. Identification of Chlamydia trachomatis DNA in human papillomavirus (HPV) positive women with normal and abnormal cytology. Arch Gynecol Obstet. 2002;266: I68-71.

I I. Smith JS, Muñoz N, Herrero R, Eluf-Neto J, Ngelangel C, Franceschi S, et al. Evidence for Chlamydia trachomatisas a human papillomavirus cofactor in the etiology of invasive cervical cancer in Brazil and the Philippines. J Infect Dis. 2002; | 85:324-3|.

12. Götz HM, Van Bergen JEAM, Veldhuijzen IK, Broer J, Hoebe CJPA, Richardus JH. A prediction rule for seletive screening of Chlamydia trachomatis infection. Sex Transm Infect. 2005;81:24-30.

13. Teles E, Hardy E, Oliveira UM, Elias CJ, Faúndes A. Reassessing risk assessment: limits to predicting reproductive tract infection in new contraceptive users. Int Fam Plan Perspect. 1997;23: 1 79-82. 
14. Mclntyre-Seltman K, Castle PE, Guido R, Schiffman M, Wheeler CM. Smoking is a risk factor for cervical intraepithelial neoplasia grade 3 among oncogenic human papillomavirus DNA-positive women with equivocal or mildly abnormal cytology. Cancer Epidemiol Biomarkers Prev. 2005; | 4: | | 65-70.

15. Ministério da Saúde. Caderno de Atenção Básica n 13. Controle dos cânceres do colo do útero e da mama. Brasília-DF; 2006. [citado $20 \mathrm{Nov}$ 2007] Disponível em: http://bvsms.saude.gov.br/bvs/publicacoes/ abcad I3.pdf.

16. Di Felice V, David S, Cappello F, Farina F, Zummo G. Is Chlamydial heat shock protein 60 a risk factor for oncogenesis? Cell Mol Life Sci. 2005;62:4-9.

17. Golijow CD, Abba MC, Mouron AS, Laguens RM, Dulout FN, Smith JS. Chlamydia trachomatis and Human papillomavirus infections in cervical disease in Argentine women. Gynecol Oncol. 2005;96: 181-6.

18. Ministério da Saúde. Programa Nacional de DST/Aids. Diagnóstico laboratorial de clamídia, $3^{a}$ ed. Brasília (DF); 1997. [citado 20 Nov 2007] Disponível em: http://bvsms.saude.gov.br/bvs/publicacoes/ cd05_09.pdf.

19. Cravioto MDC, Matamoros O, Villalobos-Zapata Y, Pena O, García-Lara E, Martinez M, et al. Prevalencia de anticuerpos anti-Chlamydia trachomatis y anti-Neisseria gonorrhoeae em grupos de indivíduos de la población mexicana. Salud Publica Mex. 2003;45:68I-9.

20. Grun L, Tassano-Smith J, Carder C, Johnson AM, Robinson A, Murray E, et al. Comparison of two methods of screening for genital Chlamydia I infection in women attending in general practice: cross sectional survey. BMJ 1997;315:226-30.
21. Molano M, Weiderpass E, Posso H, Morre AS, Ronderos M, Franceschi $\mathrm{S}$, et al. Prevalence and determinants of Chlamydia trachomatis infections in women from Bogota, Colombia. Sex Transm Infect. 2003;79:474-8.

22. Edelman M, Fox A, Alderman E, Neal W, Shapiro A, Silver EJ, et al. Cervical Papanicolaou smear abnormalites and Chlamydia trachomatis in sexually active adolescent females. I Pediatr Adolesc Gynecol. 2000;13:65-9.

23. Reesink-Peters N, Ossewaarde JM, Van Der Zee AGJ, Burger MPM, Adriaanse AH. No association of anti-Chlamydia trachomatis antibodies and severity of cervical neoplasia. Sex Transm Infect . 200 I;77: I 0 I-2.

24. Smith JS, Muñoz N, Herrero R, Eluf-Neto J, Ngelangel C, Franceschi S, et al. Evidence for Chlamydia trachomatisas a human papillomavirus cofactor in the etiology of invasive cervical cancer in Brazil and the Philippines. J Infect Dis. 2002; | 85:324-31.

25. Koskela P, Anttila T, BjÃ rge T, Brunsvig A, Dillner J, HakamaM, et al. Chlamydia trachomatisinfection as a risk factor for invasive cervical cancer. Int J Cancer. 2000;85:35-9.

26. Finan RR, Tamim H, Almawi WY. Identification of Chlamydia trachomatis DNA in human papillomavirus (HPV) positive women with normal and abnormal cytology. Arch Gynecol Obstet. 2002;266: I68-71 .

Artigo recebido: 23// I/07

Aceito para publicação: 19/04/08 